\title{
Development of Antibacterial and Thermal Storage Carbon/Silver Fabric by Using Thermoprint and Fitting Technique
}

\author{
Guifu Li ${ }^{1, a}$, Chunxia CHEN ${ }^{1, b}$,Lei Zhao ${ }^{1, c}$ \\ ${ }^{1}$ Yan cheng institute of industry technologe, Yancheng, 224005, china \\ ${ }^{a}$ Igf9928@126.com, ${ }^{b}$ chx9941@163.com, ${ }^{\text {C } z h a o l e i 7365 @ 163 . c o m ~}$
}

Keywords: Antibacterial Carbon/silver film thermal fabric; design; properties; test;

Abstract: This paper introduced an Antibacterial Carbon/silver film thermal fabric designed by using thermoprint and fitting technique, and introduced the base fabric selection 、 Product specifications 、technological process 、process parameters and key technologies detailedly,then tested antibacterial property , tear strength 、 the washing Appearance changes、abrasion resistance、warmth retention property according to the standard, Through the detection of the fabric we found that antibacterial rate of Staphylococcus aureus of the fabric up to $97.9 \%$, the warp tear strength is $13.4 \mathrm{~N}$, and the weft tear strength is $12.9 \mathrm{~N}$, after washing the Carbon/silver film face has no significant Shedding, abrasion resistance $>5000$ turns,thermal resistance is 0.013 , water- vapor resistance is 25.1 ,test data shows that the designed functional fabric meet the technology requirement of the standards, meet the demand of the customer's use requirements.

\section{Introduction}

silver pressed side

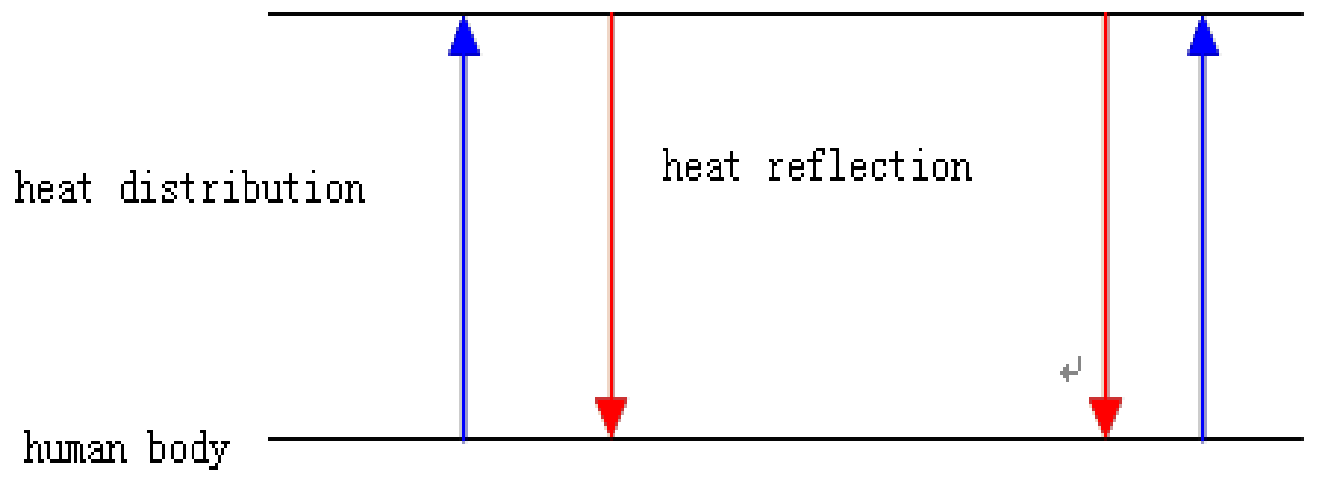

Fig.1. Heat preservation Mechanism diagram

Combination Gilding process of Printed matter we use the carbon silver film on 4-way Spandex woven fabric.It can make the fabric not only has the characteristics of heat preservation, antibacterial and deodorant, but also has the characteristics of fashion, smaller friction coefficient, wind proof and good elasticity. This product is woven fabric whose surface is coated with the activated carbon powder. Carbon powder materials is a kind of biological materials, which is non-toxic harmless. Carbon powder can be added into PU resin, which can make the fabric have the functions of moisture absorption、deodorant、antibacterial、 negative ion 、 anti ultraviolet、 heat preservation and so on. The product' PU resin was also added into silver powder. Silver powder is commonly known as the "Aluminum powder", which has the Light weight, high floating force、strong hiding power. and good reflection of light and heat. Also can made the derived textiles have the heat preservation and the metal appearance[1-3]. Fig. 1 is the heat preservation Mechanism diagram of the silver pressed fabric. 


\section{Experiment}

\section{Materials and instruments}

\section{Fabric specifications}

Fabric:4-way Spandex fabric;Linear density:75D+40D*75D+40D;

Weight: $105 \mathrm{~g} / \mathrm{m}^{2}$; Material:T(94\% ),op(6\%); Warp density: 480 roots $/ 10 \mathrm{~cm}$;Weft density:430 roots $/ 10 \mathrm{~cm}$;

Fabric weave:Plain weave

\section{Selection of transfer film substrate}

At present, the main heat transfer film substrate include OPP、PET、PVC、CP, This product used PU resin with carbon powder and silver powder to imprint with cloth through OPP transfer film, Select OPP film mainly because the materials have high smoothness and transparency, temperature resistance and Climate resistance.

\section{Equipment}

The production equipment of antibacterial fabric is provided by jiang su nanwei Yueda,The equipment consists of four parts:Glue table、 oven、 tension control device of carbon silver film, winding device and stripping device.

\section{Technological process}

\section{Process flow chart}

The process flow chart of the antibacterial carbon/silver film thermal Fabric is shown in Fig.2.

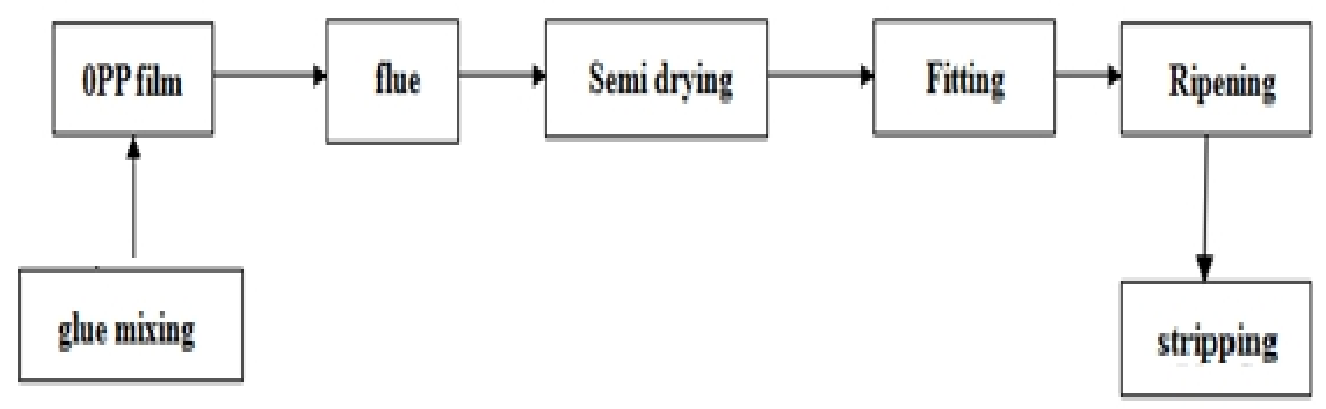

Fig.2. Process flow chart of the Antibacterial Carbon/Silver Film Thermal Fabric

\section{Fitting Technological process}

The technological process digram of the antibacterial carbon/silver film thermal Fabric is shown in Fig.3.

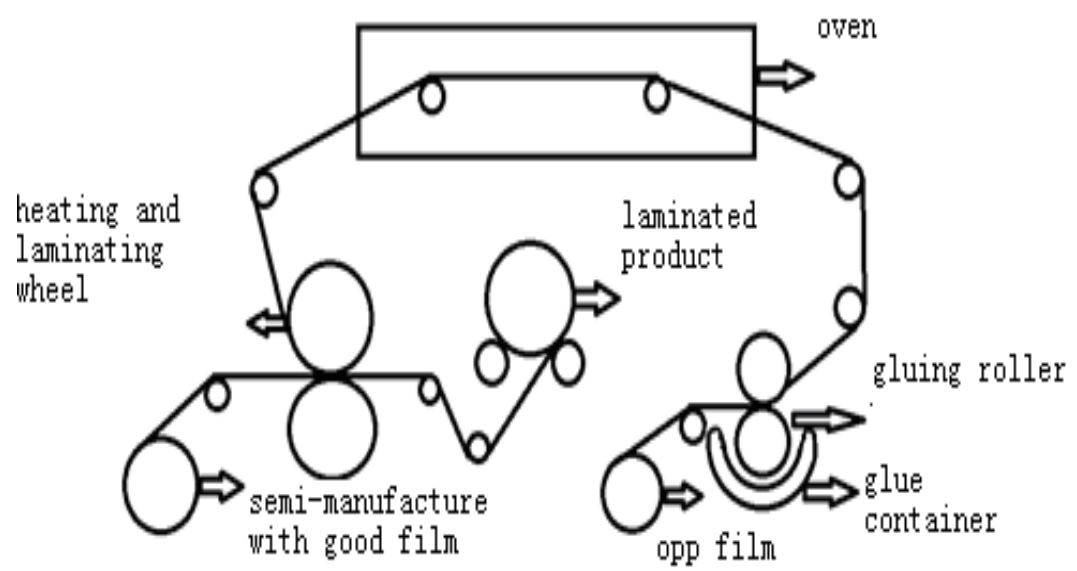

Fig.3. Technological process diagram 


\section{stripping process diagram}

The stripping process diagram of the antibacterial carbon/silver film thermal Fabric is shown in Fig.4.

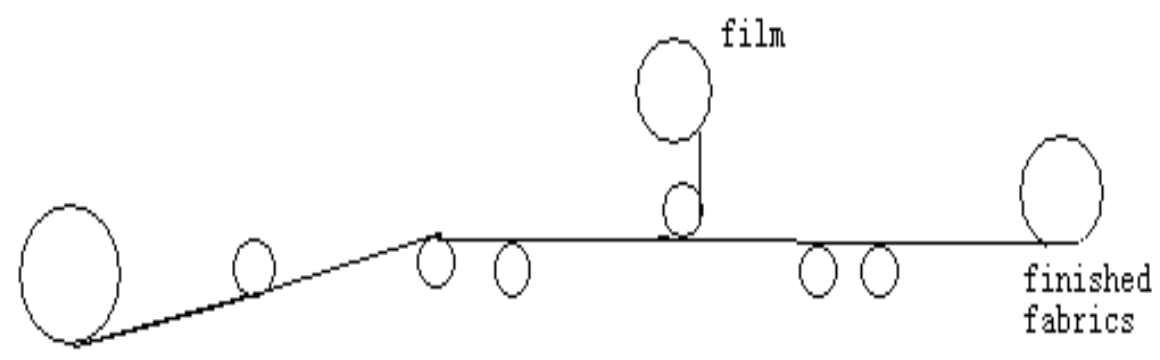

Fig.4. Stripping process diagram

\section{Place the finished fabrics in the ripening room to ripen}

\section{Process parameters and key technologies}

\section{Determination of hot stamping pattern}

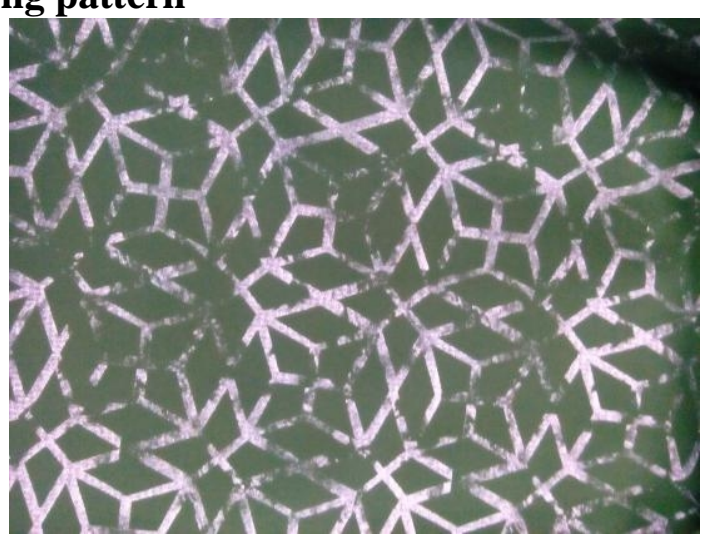

Fig.5. Face side silver pressed pattern

The pattern size determines the carbon and silver film coverage on the cloth, and also determines gluing quantity. Pattern density determines the product's insulation performance and fabric feel, Therefore, the pattern design is very important.

For this product, the face side of the woven fabric is silver pressed, the pattern is stereoscopic window grilles, the inner side of the fabric is laminated OPP film, on the film is carbon/silver pressed which with the pattern of honeycomblike. The specific pattern is shown in Fig.5 and Fig.6.

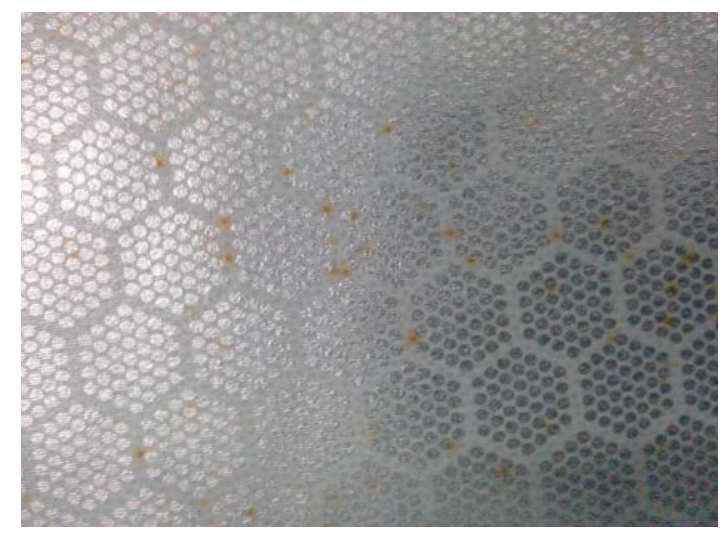

Fig.6. OPP film silver pressed pattern 


\section{Temperature determination}

The temperature mainly includes hot stamping temperature, oven temperature and fitting wheel temperature, The temperature has a great influence on the quality of hot stamping.If the temperature is too low, the melting will be insufficient, which result in no hot stamping or weakly stamping, If the temperature is too high, it will make film to melt, And also lead to the oxidation between the surface of the dyed layer and carbon silver film, so that the hot stamping products lose the metallic luster, reduce the brightness, according to the performance of the carbon silver film, hot stamping stress, hot stamping speed, for this production the hot stamping temperature range is 70 to $150^{\circ} \mathrm{C}$.Oven temperature: $40 \sim 45^{\circ} \mathrm{C}$. Fitting wheel temperature: $100 \sim 120^{\circ} \mathrm{C}$.

\section{Laminated pressure determination}

The function of the Laminated pressure, The first is to ensure the TPU film can be adhered to the substrate, Laminated process itself is the process which use the temperature and pressure to glue the fabric to the substrate. If the pressure is small, it will not be able to make the fabric adhere the substrate, which result in no laminating or weakly laminated, If the pressure is too big, the fabric and substrate 's compression deformation will increase. For this product the laminated pressure is 6 $-6.5 \mathrm{Kg}$.

\section{Speed determination for hot stamping}

The contacting time between carbon silver film and substrate be determined by the hot stamping speed, and the contacting time determines the stamping fastness, the speed must accommodate to the pressure and temperature, too fast and too slow won't be good. For this product the speed is about $15 \sim 20 \mathrm{~m} / \mathrm{min}$.

\section{Determination of tension control}

Degree of tightness for silver film tension should be appropriate, if the tension is bigger, the lines will be lack or broken.If looser the film surface will be folded to death. The cloth tension control should be appropriate too, if the tension is bigger will produce shrinkage for the silver pattern, and the pattern will broken.If looser will produce dead fold and indentation. Therefore, the cloth and film feeding tension and winding tension are extremely important.

\section{Glue formula}

The formula determines the silver pressed fastness, but the adjustment of the formula and the temperature, speed, pressure and pattern of the process are related, the choice of solvent determines the temperature and speed, choose the volatile solvents need to reduce the temperature, The ratio of the additive agent based on the pattern which determine the glue's viscosity and pressure. Therefore, it's need to determine the solvent ratio and solvent type in the glue according to the pattern. For this product, the glue formula is shown in Table.1.

Table.1 Glue formula

\begin{tabular}{cc}
\hline Reagent name & Proportion \\
\hline Polyurethane adhesive & 100 \\
MEK & 120 \\
Bridging agent & 8 \\
Silver powder & 20 \\
Carbon powder & 10 \\
Promoters & 3 \\
\hline
\end{tabular}




\section{Ripening temperature and time determination}

Ripening temperature and time determines the hot silver fastness so as to improve hot silver wash ability and soft feel. For this product the ripening temperature and time: $65^{\circ} \mathrm{C}, 12 \mathrm{~h}$.

\section{Performance test}

Table. 2 Testing Results

\begin{tabular}{|c|c|c|c|c|}
\hline Item & Standard & Index & Result & Judgement \\
\hline $\begin{array}{l}\text { Washing } \\
\text { appearance }\end{array}$ & AATCC 124-2011 & 10 laundering & 3.0 grade & up to standard \\
\hline $\begin{array}{l}\text { Colorfastness } \\
\text { to laundering }\end{array}$ & AATCC 61 & 4 and over & $\begin{array}{l}\text { Color fastness: } 4.5 \\
\text { staining color } \\
\text { fastness : } 4.5\end{array}$ & up to standard \\
\hline $\begin{array}{l}\text { Abrasion } \\
\text { resistance }\end{array}$ & ASTMD4966-2010 & OVER 5000 & $\geq 5000$ turns & up to standard \\
\hline $\begin{array}{l}\text { Tearing } \\
\text { strength }\end{array}$ & ASTMD1424-2009 & $10 \mathrm{~N}$ & $\begin{array}{ll}\text { warp: } & 13.4 \\
\text { weft: } & 12.9\end{array}$ & up to standard \\
\hline $\begin{array}{l}\text { Physiological } \\
\text { effects }\end{array}$ & GB 11048 & $\begin{array}{c}\text { Thermal } \\
\text { resistance } \geq 0.01 \\
\text { Water vapor } \\
\text { resistance } \geq 23\end{array}$ & $\begin{array}{c}0.013 \\
25\end{array}$ & up to standard \\
\hline $\begin{array}{l}\text { Antibacterial } \\
\text { test }\end{array}$ & GBT20944.3-2008 & $\begin{array}{c}\text { antibacterial rate } \\
\text { (Staphylococcu } \\
\text { s aureus ) }\end{array}$ & $97.9 \%$ & up to standard \\
\hline
\end{tabular}

The Products were produced by Jiangsu Nanwei Yueda Fiber Technology Co.Ltd and were sent to CNTAC Testing Center-Suzhou Zhongke Textile Technology Service Co.Ltd.The Test standards and the corresponding index and test results are shown in the Table.2[4-6].Testing data from Table.2 show hat the designed product's performance on antibacterial、washing appearance、 color fastness 、 wear resistance and heat preservation, strength have all reached technical requirements of the standards, meet the requirements of the normal customers, fulfill the original intention of the design[7-8].

\section{Conclusions}

Thermal storage carbon/silver fabric, use the 4-way Spandex fabric as the base cloth, using the hot stamping and fitting techniques, according to the self-designed pattern, and transferred carbon silver film to the woven fabrics.

The product Mainly were endowed with excellent antibacterial deodorant, heat preservation effect, and this product has the fashion and novel appearance、smooth、 easy to put-on- and-off and other excellent performance too.

This functional fabric is preferred fabric used as lining of wind jacket, inside, Outdoor Jackets,golf shirt and so on .

\section{Acknowledgements}

The work is supported by visiting engineers program project in higher vocational colleges of Jiangsu Province, the project number is 2014FG107. 


\section{References}

[1] YANG Na,YAN Yurong,ZHAO Yaoming AG-TYPE ANTIBACTERIAL AGENT AND ITS APPLICATION IN THE TEXTILE MATERIAL[J].CHEMICAL FIBER \& TEXTILE TECHNOLOGY,2004,9(3):20-23.

[2] XU Jia,XU Xufan. Effects of DF nano silver on waterproof and breathable and antibacterial properties of the composite fabrics[J].Silk Monthly,2012,49(2):7-10.

[3] WANG Chunmei,LI Zhao-hui. Analysis of factors affecting performances of waterproof

\&moisture permeable laminated fabric[J].SHANGHAI TEXTILE

SCIENCE\&TECHNOLIGY,2009,37(7):52-53.

[4] GB/T 11048-2008 Textiles-Physiological effects-measurement of thermal and water-vapour resistance under steady-state conditions,Beijing.

[5] AATCC124-2011 Textiles-Smoothness appearance of fabrics after repeated home laundering.

[6] ASTMD4966-2010 Textiles-standard test method for abrasion resistance of textile fabrics(martindale abrasion tester method).

[7] ASTMD1424-2009 Standard test method for tearing strength of fabrics by falling-pendulum(Elmendorf-type)apparatus.

[8] GB/T 20944-2008 Textiles-Evaluation for antibacterial activity-part 3:shake flask method[S],Beijing. 